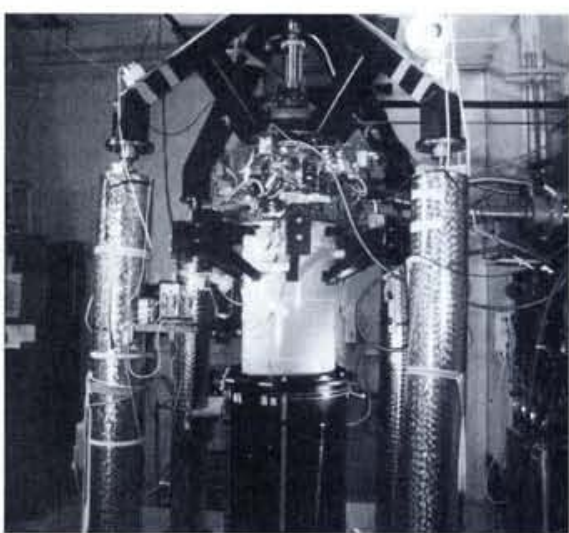

The ROTA 2 rotating liquid helium cryostat at the Helsinki University of Technology.

examples in his own field of low-temperature physics (noting that its situation is perhaps exceptional). A formal collaboration exists between the I.V. Kurchatov Institute for Atomic Energy which has large departments for low-temperature physics and technology (with 3000 staff members it is the world's largest institute in the field), the Physics Institute in Tbilissi, Georgia, his own P.L. Kapitza Institute for Physical Problems in Moscow, and the Helsinki University of Technology. The collaboration was set up to build and operate the ROTA 2 rotating liquid helium cryostat [EN 22 (1991) 3]. An agreement required the former Soviet Union to supply liquid helium but this has now become extremely difficult to obtain in the CIS. Working conditions in Tbilissi have also "become impossible". Nevertheless, the collaboration continues, and like other existing collaborations, can be expanded within the short term "without complication".

\section{East-West Coordination Committee}

The East-West Coordination Committee (EWCC) of EPS, formerly the EPS East-West Task Force, comprises:

Chairman:

A. Landesman

Les Editions de Physique

Ave. du Hoggar

Z.I. de Courtabœuf, BP 112

F-91944 Les Ullis Cédex A

Tel./Fax: + 33 (1) 69073688 / 69288491

Secretary:

E.W.A. Lingeman

NIKHEF

Postbus 4395

NL-1009 AJ Amsterdam

Tel./Fax: + 31 (20) $5922117 / 5922165$

ed@nikhefk.nikhef.nl

Members: I. Abonyi, Budapest

M. Mateev, Sofia

J. Nadrchal, Prague

O. Gherman, Craiova

J. Spalec, Warsaw

I. Slaus, Zagreb

W. Lock, CERN

Observers: G. Ripka, Saclay

J. Ziman, London

\title{
Unique Russian Training Scheme at Risk
}

\begin{abstract}
"Naive privatisation" in Russia is putting at risk a cornerstone of the country's higher education in physics. How is this? The problem concerns an unique training scheme which is relatively unknown abroad. It was set up in 1946 by P.L. Kapitza and L. Landau. Under the scheme, many Russian physics and engineering students (some 6000) aiming for research and postgraduate training are enrolled and housed at the Moscow Physical-Technical Institute (MPTI) at Dolgo Pradnaya just outside the city after a competitive entrance exam (Siberia has an equivalent institute on a large campus at Novosibirsk). The students attend basic courses given by MPTI staff during the first year. In the second year, they start spending a day a week in one of the region's 50 or so research institutes, which may belong to either a ministry or to the Russian Academy of Sciences.
\end{abstract}

Each institute has "chairs" made up of the staff members who teach students. Each chair has a chairman, and a full hierarchy of positions (head, full professor, etc.) and its members, typically 10 in number, are appointed jointly by the institute and the Council of the MPTI; some chair members also teach at the MPTI. Members are usually recommended for appointment by the MPTI based on teaching skills or by virtue of being a leader of a research group. Students apply to individual institutes which make their own selection to fill preset quota.

The time spent at the institute rises to five days each week by the fifth and sixth years when a diploma thesis is written and defended in the institute. L. Okun, a particle physicist who heads a chair in ITEP, the Institute for Theoretical and Experimental Physics, notes that some students are actively engaged in research projects by the fourth year, and that a Ph.D. can in principle be awarded only one year after the diploma (3-4 years is more normal for a theoretical thesis, and 7-8 for an experimental one).

So the system can be highly motivating, as witnessed by the many eminent Russian physicists who received their training this way. They include the present Russian Minister of Science, L.P. Gorkov (solidstate theory); M. Boloschin, A. Migdal, A. Polyakov, M. Shifman (particle physics); A. Doglov, R. Sunyaev (space, astrophysics). Well-known heads of chairs include Yu.A. Ossipyan, L.P. Gorkov, S. Belyaev, and Academician Abricosov.

The Kapitza-Landau system undoubtedly offers many advantages: young people stay close to research and grow into the research system; undergraduates have experts as teachers, enter the spirit and life of the institute, and are often selected for staff positions during their training.

But is the model viable for future? At present, the system is fairly stable, chairs included, although the number of students ap-

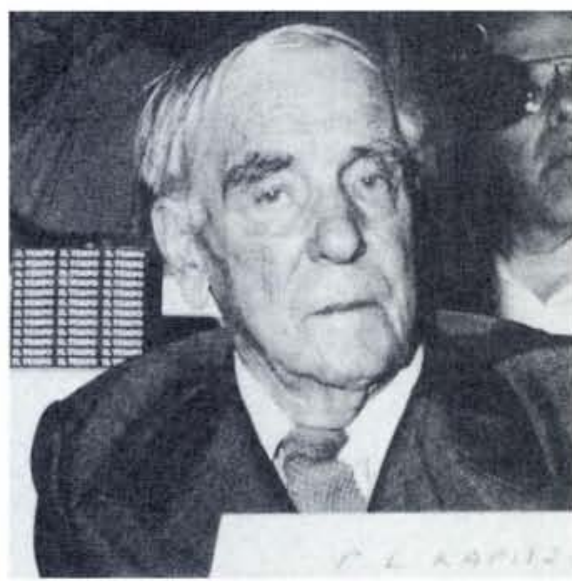

P.L. Kapitza

plying to MPTI is decreasing. More disturbing is the "privatisation" where the research institutes are increasingly expected to pay the universities and institutes other than the MPTI for the students they are sent (the MPTI remains by far the predominant partner as the universities and other institutes are only responsible for about $20 \%$ of the scheme's students). Having a large central organisation charged with teaching and no research has drawbacks, and contrasts with the large, conventional universities that have research integrated into the academic structure. These include Moscow State and St. Petersburg - each with some 20000 students spread over all facilities, not just science and engineering. The Kapitza-Landau system of research training may also seem to be elitist and focussed on a limited view of research training. But by its very success, it does at least shatter the stereotype that the only correct place to do fundamental research is in the universities.

\section{Science Policy and Management}

\section{PROCEEDINGS AVAILABLE}

The proceedings, edited by E.W.A. Lingeman, of the Europhysics Study Conference "Science Policy and Management" held at Schloss Dagstuhl, Germany, on 18-21 August 1991 can be ordered from the EPS Geneva Secretariat. The price is SFR 50. - plus postage (participants will each receive a free copy).

The proceedings represent a goldmine of information. They include invaluable and detailed presentations by representatves of nine major European research funding organisations together with analyses of general issues. 